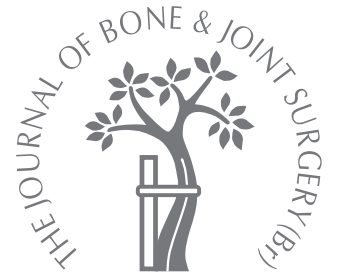

P. J. Walmsley, M. B. Kelly, J. E. Robb, I. H. Annan, D. E. Porter

From The Royal Hospital for Sick Children, Edinburgh, Scotland

P. J. Walmsley, MRCS(Ed), Specialist Registrar

M. B. Kelly, MRCS(Eng), Specialist Registrar

J. E. Robb, MD, FRCS(Ed),

Consultant

III. H. Annan, FRCS(Ed),

Consultant

D. E. Porter, MD, FRCS(Orth), Senior Lecturer

The Royal Hospital for Sick

Children, Sciennes Road,

Edinburgh EH9 1LF, UK.

Correspondence should be sent to $\mathrm{Mr}$ P. J. Walmsley at 49

Timber Bush, Leith, Edinburgh EH6 6QH, UK; e-mail: pjwalmsley@rcsed.ac.uk

(C)2006 British Editorial Society of Bone and Joint Surgery doi:10.1302/0301-620X.88B4. $17491 \$ 2.00$

$J$ Bone Joint Surg $[\mathrm{Br}]$ 2006;88-B:528-30.

Received 29 November 2005 ; Accepted 20 December 2005

\title{
Delay increases the need for open reduction of type-III supracondylar fractures of the humerus
}

\begin{abstract}
Recent reports have suggested that a delay in the management of type-III supracondylar fractures of the humerus does not affect the outcome. In this retrospective study we examined whether the timing of surgery affected peri-operative complications, or the need for open reduction. There were 171 children with a closed type-III supracondylar fracture of the humerus and no vascular compromise in our study. They were divided into two groups: those treated less than eight hours from presentation to the Accident and Emergency Department (126 children), and those treated more than eight hours from presentation (45 children). There were no differences in the rate of complications between the groups, but children waiting more than eight hours for reduction were more likely to undergo an open reduction $(33.3 \%$ vs $11.2 \%, p<0.05)$ and there was a weak correlation $(p=0.062)$ between delay in surgery and length of operating time. Consequently, we would still recommend treating these injuries at the earliest opportunity.
\end{abstract}

Supracondylar fractures of the distal humerus are the second most common fracture in children and the most frequent before seven years of age. ${ }^{1}$ Completely displaced fractures (Gartland type- $\left.-\mathrm{III}^{2}\right)$ are a challenging injury and complications include neurovascular compromise, ${ }^{2-5}$ ischaemic contracture, ${ }^{6}$ angular deformity $^{7}$ and elbow stiffness. ${ }^{8}$ Generally, type-III fractures, with or without vascular compromise, have been considered as an emergency, requiring early reduction. However, several recent publications ${ }^{9-12}$ have questioned this for closed injuries without vascular compromise and have reported no significant difference in outcome for those delayed more than eight to 12 hours. Some of the studies examined a mixture of Gartland type-IIA, type-IIB and type-III injuries. ${ }^{10,11}$ We questioned whether the management of type-III fractures should be altered in view of these reports and undertook the present study in order to identify whether a delay in management of type-III injuries affected the outcome.

\section{Patients and Methods}

The study population comprised all children with a supracondylar fracture of the humerus presenting to this hospital between August 1995 and August 2004. Open injuries and those with vascular compromise were excluded. We also excluded those who had their initial procedure outside our referral area, chil- dren with metabolic bone disease, those who did not complete follow-up, and those with ipsilateral fractures, which required treatment at the same time as the supracondylar fracture. The classification of supracondylar fractures of the humerus from lateral radiographs of the elbow described by Gartland, has been shown to have good inter- and intraobserver reliability. ${ }^{13}$

All children presented to an Accident and Emergency Department following their injury. The time of presentation noted in the Emergency Department records was recorded. We examined the ambulance records in an attempt to gain a more accurate estimate of the time of injury, but these were incomplete and therefore unreliable for the assessment of delay. Since 1992, all Emergency Departments in our catchment area have electronically recorded demographic data and the time of arrival. All children were assessed using standard protocols for their injury, given analgesia, and had their arm splinted in the position at presentation. No attempts were made to reduce the fracture before surgery and the children were taken to theatre at the earliest opportunity for reduction and percutaneous pinning of the fracture. Open reduction was performed if a closed reduction was unsuccessful. The wire configuration was either two crossed Kirschner (K-) wires or two lateral $\mathrm{K}$-wires, inserted at the surgeon's discretion. If crossed wires were 
Table I. Patient groups

\begin{tabular}{lll}
\hline & Group A (< 8 hours) & Group B (> 8 hours) \\
\hline Number & 126 & 45 \\
Mean age in yrs (range) & $6.4(1$ to 13) & 5.8 (1 to 11) \\
Male:Female & $70: 56$ & $18: 27$ \\
Left:Right & $79: 47$ & $29: 16$ \\
\hline
\end{tabular}

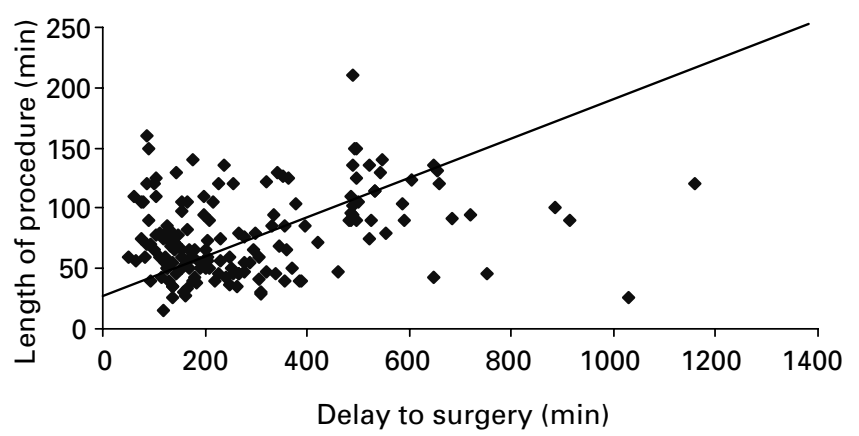

Fig. 1

Delay to surgery compared with length of procedure.

Table II. Number of complications

\begin{tabular}{lrrrc}
\hline & A & B & p value \\
\hline Closed (\%) & $112(88.8)$ & $30(66.6)$ & $<0.05$ \\
Open (\%) & $14(11.2)$ & $15(33.3)$ & $<0.05$ \\
Median nerve palsy (\%) & $13(10.3)$ & $6(13.3)$ & $\mathrm{NS}^{*}$ \\
Ulnar nerve palsy (\%) & $4(3.1)$ & $1(2.2)$ & $\mathrm{NS}$ \\
Radial nerve palsy (\%) & $5(3.9)$ & $2(4.4)$ & $\mathrm{NS}$ \\
Anterior interosseous palsy (\%) & $10(7.9)$ & $4(8.8)$ & $\mathrm{NS}$ \\
Wound infection & 1 & 1 & - \\
Cubitus varus & 5 & 3 & -
\end{tabular}

* NS, not significant

used, a mini-incision was made on the ulnar side of the fracture to safeguard the ulnar nerve. The elbow was immobilised in an above-elbow backslab, which was retained for three weeks, after which the wires were removed. All procedures were supervised or carried out by a consultant (JER, IHA, DEP, JMB, MFM).

The theatre computer log was examined and we recorded the time the patient entered theatre from the anaesthetic room, as well as the time they left theatre, in order to allow calculation of the duration of the procedure, excluding anaesthesia, and the delay between admission and surgery. The study group was then divided into those treated before and those treated after eight hours of admission. The reason for delay was recorded.

The inpatient and outpatient medical records and the children's radiographs were examined. Each child's age, gender, side of injury, pin configuration, maintenance of fracture reduction and peri-operative complications (e.g. compartment syndrome, vascular compromise, pin-track infection and iatrogenic nerve injury) were noted, as was whether an open or a closed approach was used. Any subsequent re-referrals with complications, such as persisting neurological signs and symptoms or cubitus varus deformity, were also recorded.

The peri-operative complications, delay between admission and surgery and the need for open reduction were analysed using Fisher's exact test of probability and a $p$ value of $<0.05$ was considered significant.

\section{Results}

During the study period, 534 children presented to our hospital with a supracondylar fracture of the humerus. Of these, 186 were type-III as diagnosed on radiographs by the admitting consultant. Ten children were excluded in accordance with the criteria outlined previously and five were lost to follow-up, leaving a study group of 171 . This was then divided into two further groups for analysis, based on the delay to surgery. Group A were those children who were treated less than eight hours from presentation (mean 196.15 minutes) and group B were those treated more than eight hours after presentation (mean 577.11 minutes).

The number of admissions to our unit per year remained fairly constant throughout the study, at between 14 and 24 . The numbers for 1995 and 2004 represent only part of those year's admissions, as the study period ran from August to August. There was no difference in the proportion of tertiary referrals in either group. The mechanisms of injury recorded were similar to those in other studies. ${ }^{9-12}$

Table I shows the demographic distribution of the two groups. Three children were admitted between 00:00 and 08:00 hrs, 60 between 08:00 hrs and 16:00 hrs, and 108 between 16:00 hrs and 00:00.

Figure 1 shows a scatter plot of the delay to surgery (minutes) against the duration of surgery (minutes). This shows a trend that, as the delay to surgery increased, the length of the surgical procedure also increased, particularly after a delay of more than eight hours. A Pearson correlation coefficient test was performed which gave an $r$ value of 0.2 ( $p=$ 0.062 ), suggesting only a weak correlation.

The most striking difference between the two groups is that more children in group $\mathrm{B}$ required an open reduction than in group $\mathrm{A}(33.3 \%$ vs $11.2 \%, \mathrm{p}<0.05)$ (Table II). The neurological complications were similar for both groups. One patient suffered a transient ulnar nerve palsy after wire insertion on the ulnar side of the fracture, but this resolved after six months. There were no episodes of compartment syndrome and no patient developed vascular compromise. There were two superficial wound infections around pin sites, both of which cultured Staphylococcus aureus, which was sensitive to penicillin. Both infections resolved completely after removal of the wire and treatment with oral penicillin. There were three children in group A in whom reduction was lost after surgery and who required a second 
closed reduction and wiring. Eight children (five from group A and three from group B) developed a cubitus varus deformity after fracture union. Only one patient required a corrective supracondylar osteotomy.

\section{Discussion}

There is good evidence to suggest that Gartland type-IIA and type-IIB injuries ${ }^{2}$ may be treated safely on the next scheduled operating list. ${ }^{9-12,14}$ This has the advantage of complying with guidelines on out-of-hours operating and anaesthesia. ${ }^{15}$ Recently, there have been reports ${ }^{9-12}$ suggesting that closed type-III injuries without vascular compromise can be left safely overnight until the scheduled list the following day. Our study has shown that in our centre, delay in performing definitive fixation increased the need for an open rather than a percutaneous procedure $(\mathrm{p}<0.05)$. There was also a weak correlation $(\mathrm{p}=0.062)$ between delay and increased operating time.

Mehlman et $\mathrm{al},{ }^{10}$ in their series of type-II and type-III injuries, defined delay as eight hours but did not reveal a difference in complications in the delayed group. The choice of eight hours appeared to be arbitrary. The groups in their series were of a similar size to those in our study, but their delayed group was larger. It is worth noting that both groups were a heterogeneous mix of injuries and that the incidence of type-III injuries varied between groups. Multivariate analysis of patient factors was not found to affect treatment or outcome.

The report by Iyengar et al, ${ }^{9}$ suggested that in a small series of children a delay in the definitive treatment of Gartland type-III injuries of more than eight hours did not increase the need for open reduction. Leet et $\mathrm{al}^{12}$ reviewed a larger series of type-III fractures and also found no correlation between delay to surgery and the need for open reduction, or adverse outcome. Gupta et $\mathrm{al}^{11}$ reported on 150 children who had undergone surgical treatment of supracondylar fractures and found no significant increase in peri-operative complications or the need for an open procedure, associated with a delay to surgery of more than 12 hours. However, their patients comprised heterogeneous groups containing both type-II and type-III injuries. A subset analysis of the type-III injuries was attempted, but this group was quite small. Furthermore, the fractures were splinted in 30 to $45^{\circ}$ of flexion while the child awaited reduction. In our unit, we splint the arm in the position at presentation and do not attempt to improve this.

The overall rate of open reduction reported in the literature varies from $1 \%$ to $46 \%{ }^{5,9-12,16-20}$ Our figures showed that the rate of open reduction increased from $11.2 \%$ to $33 \%$ if surgery was delayed for more than eight hours. Our results were from a single centre and included all local children in addition to tertiary referrals, which differs from other studies. All children included in our study had type-III injuries, and the procedures were supervised or managed by a consultant.

In summary, this retrospective study showed that in our unit, delaying surgery for Gartland type-III supracondylar fractures of the humerus was associated with an increased rate of open reduction. No increase in the incidence of soft tissue or neurological injury was found. As a result, despite recent reports to the contrary, ${ }^{9-12}$ we do not support the concept of delaying the management of type-III injuries.

We thank Ms Julie McBirnie and Mr Malcolm Macnicol for access to details of children that have been under their care.

No benefits in any form have been received or will be received from a commercial party related directly or indirectly to the subject of this article.

\section{References}

1. Cheng JC, Shen WY. Limb fracture patterns in different paediatric age groups: a study of 3350 children. J Orthop Trauma 1993;7:15-22.

2. Gartland JJ. Management of supracondylar fractures of the humerus in children. Surg Gynecol Obstet 1959;109:145-54.

3. Brown IC, Zinar DM. Traumatic and iatrogenic complications after supracondylar fractures of the humerus in children. J Pediatr Orthop 1995;15:440-3.

4. Copley L, Dormans J, Davidson R. Vascular injuries and their sequelae in paediatric supracondylar humeral fractures: toward a goal of prevention. J Pediatr Orthop 1996;16:99-103.

5. Cramer CE, Green NE, DeVito DP. Incidence of anterior interosseous nerve palsy in supracondylar humerus fractures in children. J Pediatr Orthop 1993;13:502-5.

6. Rang M. Children's fractures. Philadelphia: J. B. Lippincott, 1974:101.

7. Boyd DW, Aronsen DD. Supracondylar fractures of the humerus: a prospective study of percutaneous pinning. J Pediatr Orthop 1992;12:789-94.

8. Coventry MB, Henderson CC. Supracondylar fracture of the humerus: 49 cases in children. Rocky M Med J 1956;53:458-65.

9. lyengar SR, Hoffinger SA, Townsend DR. Early versus delayed reduction and pinning of type III displaced supracondylar fractures of the humerus in children: a comparative study. J Orthop Trauma 1999;13:51-5.

10. MehIman CT, Strub WM, Roy DR, Wall EJ, Crawford AH. The effect of surgical timing on the perioperative complications of supracondylar humeral fractures in children. J Bone Joint Surg [Am] 2001;83-A:323-7.

11. Gupta N, Kay RM, Leitch $\mathbf{K}$, et al. Effect of surgical delay on perioperative complications and the need for open reduction in supracondylar humerus fractures in children. J Pediatr Orthop 2004;24:245-8.

12. Leet AL, Frisancho J, Ebramzadeh $\mathbf{E}$. Delayed treatment of type 3 supracondylar humerus fractures in children. J Pediatr Orthop 2002;22:203-7.

13. Barton KL, Kaminsly CK, Green DW, et al. Reliability of the Gartland classification of supracondylar humerus fractures. J Pediatr Orthop 2001;21:27-30.

14. O'Hara LJ, Barlow JW, Clark NMP. Displaced supracondylar fractures of the humerus in children. J Bone Joint Surg [Br] 2000;82-B:204-10.

15. Who Operates When? A report by the National Confidential Enquiry into Perioperative Deaths 1995/96. London, 1997. www.ncepod.org.uk (accessed 27/11/05).

16. Danielsson L, Pettersson H. Open reduction and pin fixation of severely displaced supracondylar fractures of the humerus in children. Acta Orthop Scand 1980;51: 249-55.

17. Hart GM, Wilson DW, Arden GP. The operative management of the difficult supracondylar fracture of the humerus in the child. Injury 1977;9:30-4.

18. Mehlman CT, Crawford AH, McMillion TL, Roy DR. Operative experience of supracondylar fractures of the humerus in children: the Cincinnati experience. Acta Orthop Belg 1996;62(Suppl 1):41-50.

19. Peters CL, Scott SM, Stevens PM. Closed reduction and percutaneous pinning of displaced supracondylar humerus fractures in children: description of a new closed reduction technique for fractures with brachialis muscle entrapment. $J$ Orthop Trauma 1995;9:430-4.

20. Van Laarhoven CJ, Oosterhuis KJ. Operative treatment of supracondylar fractures of the humerus in children. Neth J Surg 1990;42:61-2. 